DR. SILVIA SOOKOIAN (Orcid ID : 0000-0001-5929-5470)

Article type : Original

This article has been accepted for publication and undergone full peer review but has not been through the copyediting, typesetting, pagination and proofreading process, which may lead to differences between this version and the Version of Record. Please cite this article as doi: $\underline{10.1111 / \text { JOIM.13147 }}$

This article is protected by copyright. All rights reserved 


\section{Liver mitochondrial DNA damage and genetic variability of Cytochrome b - a key component of the respirasome - drive the severity of fatty liver disease}

Short title: NASH and respirasome

Carlos J Pirola *, Martin Garaycoechea, Diego Flichman, Gustavo O Castaño and Silvia Sookoian

${ }^{1}$ University of Buenos Aires, School of Medicine, Institute of Medical Research A Lanari, Ciudad Autónoma de Buenos Aires, Argentina (CJP, SS).

${ }^{2}$ National Scientific and Technical Research Council (CONICET)-University of Buenos Aires, Institute of Medical Research (IDIM), Department of Molecular Genetics and Biology of Complex Diseases, Ciudad Autónoma de Buenos Aires, Argentina (CJP).

${ }^{3}$ Department of Surgery, Hospital de Alta Complejidad en Red "El Cruce”, Florencio Varela, Buenos Aires, Argentina (MG).

${ }^{4}$ Department of Virology, School of Pharmacy and Biochemistry, University of Buenos Aires, Ciudad Autónoma de Buenos Aires, Argentina (DF).

${ }^{5}$ Liver Unit, Medicine and Surgery Department, Hospital Abel Zubizarreta, Ciudad Autónoma de Buenos Aires, Argentina (GOC).

${ }^{6}$ National Scientific and Technical Research Council (CONICET)-University of Buenos Aires, Institute of Medical Research (IDIM), Department of Clinical and Molecular Hepatology, Ciudad Autónoma de Buenos Aires, Argentina (SS).

${ }^{*}$ Co-corresponding authorship

Grant support: This study was partially supported by grants PICT 2015-0551 and PICT 20160135 (Agencia Nacional de Promoción Científica y Tecnológica, FONCyT). CONICET Proyectos Unidades Ejecutoras 2017, PUE 0055.

\section{Abbreviations:}

ALT, alanine-aminotransferase

AST, aspartate-aminotransferase

BMI, body mass index

MT-CYB, mitochondrially encoded cytochrome b

HOMA, homeostasis model assessment

MtDNA, mitochondrial DNA

This article is protected by copyright. All rights reserved 
MT-CYB, mitochondrially encoded cytochrome b

NAFL, nonalcoholic fatty liver

NAFLD, nonalcoholic fatty liver disease

NASH, nonalcoholic steatohepatitis

OXPHOS, oxidative phosphorylation

ROS, reactive oxygen species

8-OHdG, 8-hydroxy-2'-deoxyguanosine

4-HNE, 4-hydroxynonenal

\section{Co-Corresponding Authors}

Carlos J. Pirola, PhD and Silvia Sookoian, MD, PhD

Instituto de Investigaciones Médicas, IDIM-CONICET

Combatientes de Malvinas 3150, CABA-1427, Argentina

E-mail: pirola.carlos@conicet.gov.ar and ssookoian@intramed.net

Phone: 54-11-52873905

CJP and SS should be considered joint senior authors

This article is protected by copyright. All rights reserved 


\section{ABSTRACT}

Background \& Aims: The progression of nonalcoholic fatty liver disease (NAFLD) into severe histological forms (steatohepatitis-NASH) is paralleled by the occurrence of complex molecular processes. Mitochondrial dysfunction is a hallmark feature of advanced disease. Mitochondriallyencoded cytochrome B (cytochrome b, $M T-C Y B$ ) - a member of the oxidative phosphorylation system, is a key component of the respirasome supercomplex. Here, we hypothesized that NAFLD severity is associated with liver tissue cytochrome $\mathrm{b}$ mutations and damaged mitochondrial DNA (mtDNA).

Methods: We included 252 liver specimens of NAFLD patients - in whom histological disease ranged from mild to severe-which were linked to clinical and biochemical information. Tissue molecular explorations included $M T-C Y B$ sequencing and analysis of differential mtDNA damage. Profiling of circulating Krebs cycle metabolites and global liver transcriptome was performed in a subsample of patients. Tissue levels of 4-hydroxynonenal-a product of lipid peroxidation and 8hydroxy-2'-deoxyguanosine and a marker of oxidative damage-were measured.

Results: Compared to simple steatosis, NASH is associated with a higher level of MT-CYB variance, 12.1 vs. 15.6 substitutions per $10^{3} \mathrm{bp}(p=5.5 \mathrm{e}-10)$. The burden of variants was associated with increased levels of 2-hydroxyglutarate, branched-chain amino acids, and glutamate, and changes in the global liver transcriptome. Liver mtDNA damage was associated with advanced disease and inflammation. NAFLD severity was associated with increased tissue levels of DNA oxidative adducts and lipid peroxyl radicals.

Conclusion: NASH is associated with genetic alterations of the liver cellular respirasome, including high cytochrome $\mathrm{b}$ variation and mtDNA damage, which may result in broad cellular effects.

Keywords: NASH; genetics; mitochondrial DNA; mutations; fibrosis; heritability; mitochondrial damage; MT-CYB; cytochrome b; respirasome; transcriptome; oxidative damage; complex III.

This article is protected by copyright. All rights reserved 


\section{INTRODUCTION}

The progression of nonalcoholic fatty liver disease (NAFLD) into severe histological forms is paralleled by the occurrence of complex molecular processes that lead to a gradual loss of organ function and systemic metabolic derangement. Liver mitochondrial structural defects and mitochondrial dysfunction are the hallmark features of advanced disease [1-6]. Nonalcoholic steatohepatitis (NASH) - the distinctive severe histological disease form marked by the presence of fatty infiltration with chronic inflammation, ballooning degeneration, and different degrees of fibrosis [7] —is associated with molecular alterations of the liver mitochondrial DNA (mtDNA), including epigenetic modifications [3] and complex mitochondrial genomes with high mutational rate and degree of heteroplasmy [6]. We previously demonstrated that the liver of NASH patients presents a high variation rate in genes of the five oxidative phosphorylation (OXPHOS) protein complexes, i.e., respiratory complexes (Complex I-IV) and ATP synthase Complex V [6]. The OXPHOS system is the main source of cell energy, as the generated electrons and protons flow across the mitochondrial membrane. This proton gradient is used for ATP synthesis. All aforementioned components of the OXPHOS complex participate in the cellular respiratory process, but four of the OXPHOS protein complexes (I to IV) associate into stable entities called respiratory supercomplexes [8,9]. In mammalian mitochondria, almost the entire Complex I is coupled with Complex III and up to four copies of Complex IV, and this network of respiratory chain complexes is known as respirasome [9]. These supercomplexes exhibit a stabilizing role in each sub-module of the respiratory complex [10], and the mitochondrially-encoded cytochrome B (cytochrome b, MT-CYB) —also known as Complex III Subunit III or cytochrome b-c1 complex or ubiquinol-cytochrome c reductase complex, is a key component of the respirasome [8,9]. Mutations of the MT-CYB gene and/or mtDNA damage at the cytochrome $\mathrm{b}$ region may lead to severe respirasome supercomplex deficiency, and eventually to cell death in tissues that require abundant energy supply $[8,9,11]$. Reactive oxygen species (ROS)-promoting mutations in Complex III have also been implicated in a range of metabolic disorders [11].

Here, we hypothesized that NAFLD severity may be driven by the accumulation of genetic alterations of the mitochondrially-encoded cytochrome $b$ within the liver tissue, including a high mutation rate and the presence of mtDNA lesions. We reasoned that exploring tissue effects across different histological stages of disease severity, as well as diverse phenotypic environments, would allow us to understand the biological implications of the impaired respirasome in NAFLD.

This article is protected by copyright. All rights reserved 
Therefore, we examined 252 liver specimens linked to clinical and biochemical information. We further sequenced the $M T-C Y B$ gene, and we explored the presence of tissue-damaged mtDNA. In addition, we assessed the potential global metabolic effects of these molecular changes.

\section{SUBJECTS AND METHODS}

\section{Study design and patient selection criteria, and physical, anthropometric, and biochemical} evaluation

Participants were selected from two different hospital-based settings, including a cross-sectional study of adult overweight/obese individuals patients diagnosed with NAFLD and features of the Metabolic Syndrome (MetS) in the Liver Unit, Hospital Abel Zubizarreta, Buenos Aires, Argentina, and an independent cohort of adult morbidly obese patients with NAFLD that underwent bariatric surgery in the Surgery Department, Hospital de Alta Complejidad en Red El Cruce, Buenos Aires, Argentina.

Inclusion criteria: patients were considered for inclusion only if they presented: 1) histopathological evidence of NAFLD, either nonalcoholic fatty liver (NAFL) or NASH, based on a liver biopsy conducted within the study period, and 2) available DNA isolated from the liver tissue to perform molecular explorations as explained in the Supplementary Appendix, which was the limiting inclusion factor.

Overweight/obese individuals patients diagnosed with NAFLD were offered a percutaneous liver biopsy if they show either abnormal aminotransferase levels (alanine and/or aspartate aminotransferase defined as $>41 \mathrm{U} / \mathrm{L}$ ), gamma-glutamyl-transferase $>50 \mathrm{U} / \mathrm{L}$, alkaline phosphatase $>250 \mathrm{UI} / \mathrm{L}$, and/or severe insulin resistance (HOMA-IR value greater than 3 ). In the case of morbidly obese cohort (bariatric surgery cohort), surgically excised liver samples were performed to all consecutive patients attending the Surgery Department, Hospital de Alta Complejidad en Red El Cruce.

This article is protected by copyright. All rights reserved 
Liver specimens of patients without NAFLD were included as a part of the molecular explorations. Further details can be found in the Supplementary Appendix.

All participants (NAFLD patients and non-NAFLD patients) were selected during the same study period from the same population of patients attending the Liver Unit, and all of them share the same demographic characteristics (occupation, educational level, place of residence, and ethnicity). Adopting a case-control design, we ensured that the control liver samples matched patients' features in each cohort (morbidly obese and non-morbidly obese).

The exclusion criteria were secondary causes of steatosis, including alcohol abuse $(\geq 30 \mathrm{~g}$ alcohol daily for men and $\geq 20 \mathrm{~g}$ for women), total parenteral nutrition, hepatitis $\mathrm{B}$ and hepatitis $\mathrm{C}$ virus infection, and the use of drugs known to precipitate steatosis. By using standard clinical and laboratory evaluation, as well as liver biopsy features when applicable, autoimmune liver disease, metabolic liver disease, Wilson's disease, and $\alpha$-1-antitrypsin deficiency were likewise ruled out in all patients.

See cohorts, participants, and analyses flow chart in Supplementary Figure 1. Biological samples from all participants were obtained with written informed consent following Institutional Review Board-approved protocols (protocol number: 104/HGAZ/09, 89/100, and 1204/2012). All the investigations performed in this study were conducted in accordance with the guidelines of the 1975 Declaration of Helsinki. Complete details regarding liver biopsy and histopathological evaluation, immunohistochemistry (IHQ), $M T-C Y B$ sequencing, tissue $M T-C Y B$ damage, serumtargeted TCA cycle metabolite profiling, gene expression profiling of the whole liver transcriptome and enrichment analysis, and statistical analysis are provided in the Supplementary Appendix. The primer sequences are shown in Supplementary Table 1 and 2.

\section{RESULTS}

The clinical and biochemical features of NAFLD and non-NAFLD patients are shown in Table 1 and Supplementary Table 3, respectively. The comparison between non-morbidly obese patients with NAFLD (NAFL and NASH), among whom histological features were obtained by percutaneous liver biopsy versus morbidly obese patients with NAFLD (NAFL and NASH), of whom histological features were obtained by surgical biopsy during bariatric surgery is shown in Supplementary Table 4.

The liver of patients with NASH exhibits high level of MT-CYB variance

This article is protected by copyright. All rights reserved 
Analysis of nucleotide changes in the amplified $M T-C Y B$ gene fragment per each individual enrolled in this study was performed based on the alignment with the reference sequence (NC_012920.1 Reference assembly). Median number of cumulative MT-CYB variants/mutations in the liver tissue (median 10, mean 12.56, range 5-66) was further used to perform the analysis of dichotomous phenotypes, such as high and low cumulative number of $M T-C Y B$ variants and variables of interest. Supplementary Figure 2 shows representative sequence electropherograms sequenced by Sanger as an example.

The comparison of $M T-C Y B$ genetic variability in the liver of NAFL (12.1 variants per $1000 \mathrm{bp}$ ) vs. NASH patients (15.6 variants per $1000 \mathrm{bp}$ ) showed that the disease severity is associated with a high mutation/variation ( $p=5.5 \mathrm{e}-10)$ rate; cumulative OR 1.30, 95\% CI: $1.19-1.41$. Likewise, analysis of liver $M T-C Y B$ sequencing revealed that the cumulative number of variants was associated with histological features, including the histological degree of steatosis $(p=3.0 \mathrm{e}-4)$, the lobular inflammation score $(p=8.0 \mathrm{e}-3)$, and the NAS score $(p=3.0 \mathrm{e}-2)$. Besides, cumulative number of $M T-C Y B$ mutations in liver tissue was associated with aminotransferases (ALT $p=$ 1.9e-2 and AST $p=1.5 \mathrm{e}-2)$, alkaline phophatase $(p=1.0 \mathrm{e}-10)$, and lactic acid $(p=2.2 \mathrm{e}-2)$ levels. We further explored phenotypic features that may influence the genetic diversity of $M T-C Y B$ and found an inverse relationship between the cumulative number of $M T-C Y B$ variants and body mass index (BMI) (Spearman R -0.40, $p=1.0 \mathrm{e}-10)$. The number of cumulative variants in the liver of morbidly obese NAFLD patients (either NAFL or NASH) was significantly lower than that obtained for NAFLD overweight/obese individuals (Post-hoc test for linear trend $p=0.0007$ Slope -1.04). The number of $M T-C Y B$ cumulative variants according to BMI status (morbidly obese vs. overweight/obese patients) and stages of the disease severity is shown in Supplementary Figure 3. No association was found between the cumulative number of $M T-C Y B$ variants and fasting glucose, insulin, HOMA-IR, and hemoglobin A1c. Complete details for every MT-CYB variant, including nucleotide position, reference SNP (rsid) identification number, alleles, gene consequence, PolyPhen prediction (if available), SIFT prediction (if available), and clinical significance (if available) are shown in Supplementary Table 5.

\section{Rare mutations reported as pathogenic}

Using the sequencing approach described above, we searched for rare-either homoplasmic or heteroplasmic $M T-C Y B$ - mutations already reported as pathogenic in the MITOMAP (http://www.mitomap.org), OMIN (Online Mendelian Inheritance in Man), and/or CLINVAR (https://www.ncbi.nlm.nih.gov/clinvar) databases. We found four rare heteroplasmic mutations- 
three missense (m.G14846A, m.G15092A, and m.T15237C) and one nonsense- stop gained (m.G15242A) - and one rare (m.G15077A) homoplasmic mutation (Figure 1AB and

Supplementary Table 6). In previous studies, these mutations were shown to impair the enzymatic function of the respiratory-chain cytochrome b protein (Supplementary Table 6). The patient carrying the homoplasmic m.G15077A mutation was a 33-year-old woman presenting with NAFLD and severe (BMI 53) obesity. None of the patients in this series had symptoms of neuromuscular and/or extra-hepatic disorders, except for the NASH patient carrying the heteroplasmic stop gained m.15242 mutation, who presented with progressive muscle weakness and pain, recurrent headaches, and vomiting. Liver MT-CYB protein expression levels in the patient carrying the nonsense mutation were severely decreased (Figure 1C).

Tissue-damaged mtDNA at cytochrome b region occurred more often among severely obese patients with advanced disease

DNA damage or DNA fragmentation may reduce the amount of amplifiable templates; therefore, sequencing of fragmented DNA might result in loss of damaged DNA sites and might induce amplification bias in such a way that the sequenced fragments contain missing or underrepresented loci information [12]. Therefore, we reasoned that the inverse association between the cumulative number of $M T-C Y B$ variants in the liver tissue and BMI might be explained by the presence of lesions and/or breaks in the mtDNA. To test this hypothesis, we assessed the presence of liver MT$C Y B$ damage by a validated quantitative-PCR method, which relies on the relative amplification of a short and a long fragment in the same region. We found that long and short fragments were amplified with comparable efficiencies, 2.064 and 1.954, respectively.

The analysis of cytochrome $b$ lesions revealed a significant and positive association between liver $M T-C Y B$ damage and BMI $(p=7.9 \mathrm{e}-5)$; logistic regression OR for mtDNA damage over the median: $1.0495 \%$ CI: $1.02-1.07, p=6.6 \mathrm{e}-4$. In addition, the differential level of $M T-C Y B$ damage, which was more pronounced in the group of morbidly obese patients, was significantly associated with the disease severity ( $p=1.0 \mathrm{e}-3)$ (Figure 2A). There was a significant $(p=4.9 \mathrm{e}-2)$ interaction between disease severity (NASH vs. NAFL) and population sample (overweight/moderately obese vs. morbidly obese patients), even after adjusting for age, sex, and HOMA index.

Liver $M T-C Y B$ damage was also significantly associated with lobular inflammation $(p=6.5 \mathrm{e}-3)$. This association remained significant in the group of morbidly obese NAFLD patients $(p=1.0 \mathrm{e}-3)$ after adjusting for age, sex, and HOMA, which was expected, as there was no association between 
the presence of liver $M T-C Y B$ damage and age, sex, history of type 2 diabetes, and/or any biochemical parameters, including glucose, insulin, or lipid-related traits.

\section{The burden of liver MT-CYB variants is associated with circulating levels of metabolites}

In a subsample of patients $(n=22)$, we explored the circulating level of Krebs cycle metabolites to test the hypothesis that the increased number of liver mutations in a component of the cellular respirasome supercomplex might induce global metabolic changes. We classified NAFLD patients into two groups based on the median cumulative number of $M T-C Y B$ variations $(<10$ and $\geq 10)$. We found that the burden of liver $M T-C Y B$ diversity was significantly associated with circulating levels of 2-hydroxyglutarate, branched-chain amino acids, and glutamate, among other metabolites (Figure 2B).

NAFLD severity is associated with increased tissue levels of DNA oxidative adducts and lipid peroxyl radicals

We hypothesized that the liver tissue of patients with more severe histological disease presents increased levels of products of oxidative damage and lipid peroxidation, which would make mtDNA more susceptible to damage and mutation. To test this hypothesis, we measured 8-OHdG and 4-HNE levels by IHQ in the tissue samples of patients showing the full spectrum of NAFLD severity, as well as in the liver tissue of patients without evidence of NAFLD.

8-OHdG immunoreactivity product was significantly associated with the disease severity (linear test for trend $p<0.0001$ ) (Figure 3A-C and Supplementary Figure 4A). Specifically, 8-OHdG immunostaining level was detected at the highest levels in the liver of NASH patients (Figure 3A), whereas it was low in the liver of NAFL patients (Figure 3B) and negligible in control (nonNAFLD) tissue samples (Figure 3C). 8-OHdG-positive immunostaining was seen at both nuclear and cytoplasmic localization, which is expected because the 8-OHdG detection method stains nuclear DNA and mtDNA as well. Likewise, we found that the cumulative number of $M T-C Y B$ mutations was significantly and positively correlated with tissue levels of 8 -OHdG $(\mathrm{R} 0.86, p=$ 4.0e-5).

Furthermore, we found that, compared with NAFL and non-NAFLD, tissue levels of 4-HNE were significantly higher in patients with NASH (Figure 3D-F and Supplementary Figure 4B). 4HNE immunoreactivity product was significantly increased in a disease-severity grade-dependent manner from non-NAFLD (control) to NAFL to NASH (linear test for trend $p=0.0007$ ), as shown Figure 3D-F. 4-HNE immunostaining was exclusively localized in the cell cytoplasm, including hepatocytes and areas of active lobular and portal inflammation, suggesting that detectable levels

This article is protected by copyright. All rights reserved 
of 4-HNE are also present in inflammatory cells (Figure 3D). Moreover, the cumulative number of MT-CYB mutations was significantly and positively correlated with tissue levels of 4-HNE (R $0.66, p=2.0 \mathrm{e}-2)$.

\section{Impact of the cumulative number of MT-CYB mutations/variation on the whole liver}

\section{transcriptome}

In view of the numerous roles that mitochondria play in metabolically active tissues, we reasoned that multiple mutations in a member of the respirasome supercomplex would be associated with broad perturbations of cellular physiology, including changes in the full range of gene expression. To assess the difference in gene expression according to the cumulative number of liver $M T-C Y B$ mutations, we explored the entire liver transcriptome.

The comparison between tissue samples of NAFLD patients with the highest cumulative number of $M T-C Y B$ mutations and the reference group (low number of cumulative mutations) showed 3291 up-regulated and 1817 down-regulated transcripts with a FDR $>0.01$. The most significantly differentially expressed genes are shown in Supplementary Table S7. Overrepresentation analysis (ORA) of genes exhibiting significantly differential expression was carried out to identify the biological processes and KEGG pathways most severely affected by the high MT-CYB mutational profile. The analysis based upon significantly up-regulated transcripts showed enrichment of pathways associated with epigenetic (chromatin and chromosome organization) and post-translational (serine phosphorylation) modifications, regulation of intracellular signal transduction, and cell cycle and migration (Figure 4 and Supplementary Table S8). Analysis based upon significantly down-regulated transcripts showed the enrichment of expected processes affected by OXPHOS chain failure, such as striated muscle contraction, as well as many critical metabolic processes, including cholesterol biosynthesis, metabolism of steroids, drug response, organic hydroxy compound metabolic process, and secondary alcohol metabolic process, which includes isocitrate (an important intermediate in the TCA), ergosterol (the most important of the D provitamins that is converted to vitamin D2 upon irradiation with UV light) and vitamin D3 metabolic processes (Figure 5 and Supplementary Table S9).

\section{DISCUSSION}

Complex III of the mitochondrial respiratory chain is central to the cellular energy production process. It is also the main site of ROS production, which may lead to high level of DNA damage. In this work, we described the association of histological features of NAFLD severity with the 
cumulative number of variants and mtDNA lesions of the mitochondrially-encoded cytochrome $b$ gene. The most remarkable feature of the present study is that molecular explorations were performed in a relatively large collection of tissue samples of NAFLD patients, which allowed us to infer a direct impact on the disease. We found that NASH, as well as features of the disease severity, including severe inflammation, were associated with high $M T-C Y B$ mutation/variation rate in the liver. The potential detrimental consequences of the high liver cytochrome $b$ gene mutation rate seem not to be limited to the severity of histological features, as it might also affect global metabolic functioning. In the analyzed samples, the burden of $M T-C Y B$ diversity was significantly associated with circulating levels of alpha-ketoglutarate/pyruvate ratio, branchedchain amino acids, glutamic acid, and 2-hydroxyglutaric acid, which are known predictors of metabolic risk $[13,14]$ and hepatic carcinogenesis [15]. Furthermore, we found that the cumulative number of $M T-C Y B$ variants was inversely correlated with BMI, which may be explained by the presence of increased mtDNA damage in morbidly obese patients.

Perturbations of mtDNA integrity and increased mutations in the mtDNA are probable consequences of oxidative injury. We found significantly increased tissue levels of DNA oxidative adducts (8-OHdG) and lipid peroxyl radicals (4-HNE). Our results are supported by earlier observations showing that (i) ROS [16] and lipid peroxidation products [17] can damage mtDNA via oxidation of guanosine to $8-\mathrm{OHdG}$ and release of 4-HNE - one of the most bioactive lipid alkenals, respectively; (ii) oxidative stress-induced lesions in mtDNA can lead to somatic mutations that accumulate, affect the integrity of respiratory chain, and cause mitochondriadependent degenerative diseases and cancer [16,18]; (iii) production of $8-\mathrm{OHdG}$ is also an indicator of the presence of single-strand breaks [19]; and (iv) mitochondria are regarded as the major cellular site for ROS and 4-HNE formation [20], the latter presumably by oxidation of abundant PUFA-containing lipids [18]. It has also been suggested that mtDNA may serve as a sentinel molecule linking oxidative damage to inflammation in parenchymal cells [21]. Seki and coworkers reported that oxidative cellular damage occurs frequently in NASH, positing that it may be associated with liver fibrosis and possibly hepatocarcinogenesis [22]. Likewise, results yielded by experimental and human studies showed that the release of mitochondrial DNA molecules to the cytosol or the extracellular space triggers a pro-inflammatory response [23]. Finally, we found that the high $M T-C Y B$ mutation rate was associated with a wide range of perturbations in pathways that are linked to cancer development and metabolic deregulation. Enrichment analysis of a set of up-regulated genes identified pathways and biological processes 
associated with epigenetic modifications and perturbations of cell cycle and migration, which is consistent with the concept that primary impairment of mitochondrial function via mtDNA mutations is a pervasive feature of cancer [24]. The enrichment analysis of a set of down-regulated genes identified pathways associated with cholesterol biosynthesis, particularly the transformation of zymosterol into cholesterol, which serves as the source of the 7-dehydrocholesterol for the synthesis of vitamin D.

It is worth emphasizing that mutations in any member of the OXPHOS chain can affect the functionality and operational capacity of the overall respiratory chain because the OXPHOS operates as one functional unit [25]. In fact, we have demonstrated that the expression of OXPHOS genes in the liver tissue is highly correlated [6].

It is also noteworthy that we found five likely pathogenic - either heteroplasmic or homoplasmic - MT-CYB mutations. To our knowledge, this is the first report concerning pathogenic mutations in this locus associated with NAFLD.

Each of the rare mutations reported here is reported as associated with multisystemic disorders, such as mitochondrial encephalomyopathy, stroke-like episodes (the MELAS syndrome), maternally inherited or aminoglycoside-induced deafness, exercise intolerance, cardiomyopathy, and/or liver failure [26-28]. Hitherto, mitochondrial hepatopathies have been regarded as primarily occurring in early childhood [27], with some of the affected children presenting with hepatic steatosis [26,27].

Our results suggest that NAFLD in patients carrying respiratory-chain Complex III mutations in the liver tissue might be regarded as a mitochondrial hepatopathy. This is consistent with the notion that mutations in the mitochondrial genome may lead to progressive chronic degenerative processes, including tissue aging and organ damage. However, direct confirmation of pathogenesis on mtDNA at the cell and/or tissue level is needed to fully understand the impact of the above mentioned observations on NAFLD disease severity. The nature of our study, which included biological samples obtained by an invasive method (liver biopsy), justifies the methodological gap that exists between cause and effect in our findings. Isolation of mitochondria from fresh liver tissue to perform, for example, blue-native polyacrylamide gel electrophoresis for supercomplex analyses is technically challenging in human biological samples because it demands considerable amount of tissue.

\section{Limitations of the Study}

This article is protected by copyright. All rights reserved 
Although our results based on the transcriptome and metabolic profiling are exploratory given the modest sample sizes on which the sub-studies were performed, there is remarkable consistency in the observations yielded, suggesting that an increased number of tissue mutations in the mitochondrial cytochrome b promotes a metabolic and cellular environment that generates oncogenic metabolites and initiates tumorigenesis. This mechanism might explain the development of de novo hepatocellular carcinoma in NASH patients. Nevertheless, future investigations will be needed to further explore the possibility of causal relationships among our findings.

In summary, much progress has been made to understand the NASH pathogenesis. Disease mechanisms involving loss of mtDNA integrity are critical not only because mtDNA is essential for mitochondrial function maintenance, but also because mtDNA regulates cell physiology. Our results highlight a novel pathogenic mechanism for understanding NAFLD severity, which may occur as the consequence of genetic alterations of the liver cellular respirasome, including respiratory-chain Complex III genetic instability and mtDNA damage. The impact of MT-CYB genetic variability on NAFLD severity seems not to be related to only the number of variants in $M T-C Y B$, but to the cumulative effects on the disease states.

Ultimately, NAFLD may exhibit the whole spectrum of a complex disease, from Mendelian syndrome-associated, to the classical polygenic and multifactorial and mitochondrial forms.

\section{Conflict of interest:}

Nothing to declare.

\section{Authors' contributions:}

CJP and SS designed research (project conception, development of overall research plan, and study oversight); CJP, DF, SS conducted research (hands-on conduct of the experiments and data collection); CJP, SS analyzed data or performed statistical analysis; CJP, MG, GOC, SS performed liver biopsies and/or collected biological specimens; CJP and SS wrote paper; CJP and SS had primary responsibility for final content.

This article is protected by copyright. All rights reserved 


\section{References}

[1] Caldwell SH, de Freitas LA, Park SH, et al. Intramitochondrial crystalline inclusions in nonalcoholic steatohepatitis. Hepatology 2009; 49(6): 1888-95.

[2] Koliaki C, Szendroedi J, Kaul K, et al. Adaptation of hepatic mitochondrial function in humans with non-alcoholic Fatty liver is lost in steatohepatitis. Cell Metab 2015; 21(5): 739-46.

[3] Pirola CJ, Gianotti TF, Burgueno AL, et al. Epigenetic modification of liver mitochondrial DNA is associated with histological severity of nonalcoholic fatty liver disease. Gut 2013; 62(9): 1356-63.

[4] Sanyal AJ, Campbell-Sargent C, Mirshahi F, et al. Nonalcoholic steatohepatitis: association of insulin resistance and mitochondrial abnormalities. Gastroenterology 2001; 120(5): 1183-92.

[5] Sookoian S, Rosselli MS, Gemma C, et al. Epigenetic regulation of insulin resistance in nonalcoholic fatty liver disease: impact of liver methylation of the peroxisome proliferatoractivated receptor gamma coactivator 1alpha promoter. Hepatology 2010; 52(6): 19922000.

[6] Sookoian S, Flichman D, Scian R, et al. Mitochondrial genome architecture in nonalcoholic fatty liver disease. J Pathol 2016; 240(4): 437-49.

[7] Brunt EM, Wong VW, Nobili V, et al. Nonalcoholic fatty liver disease. Nat Rev Dis Primers 2015; 1: 15080 .

[8] Chaban Y, Boekema EJ, Dudkina NV. Structures of mitochondrial oxidative phosphorylation supercomplexes and mechanisms for their stabilisation. Biochim Biophys Acta 2014; 1837(4): 418-26.

[9] Schagger H, Pfeiffer K. Supercomplexes in the respiratory chains of yeast and mammalian mitochondria. EMBO J 2000; 19(8): 1777-83. 
[10] Lobo-Jarne T, Ugalde C. Respiratory chain supercomplexes: Structures, function and biogenesis. Semin Cell Dev Biol 2018; 76: 179-90.

[11] Acin-Perez R, Bayona-Bafaluy MP, Fernandez-Silva P, et al. Respiratory complex III is required to maintain complex I in mammalian mitochondria. Mol Cell 2004; 13(6): 805-15.

[12] Baumer C, Fisch E, Wedler H, Reinecke F, Korfhage C. Exploring DNA quality of single cells for genome analysis with simultaneous whole-genome amplification. Sci Rep 2018; 8(1): 7476.

[13] Sookoian S, Castano GO, Scian R, et al. Serum aminotransferases in nonalcoholic fatty liver disease are a signature of liver metabolic perturbations at the amino acid and Krebs cycle level. Am J Clin Nutr 2016; 103(2): 422-34.

[14] Yin X, Subramanian S, Willinger CM, et al. Metabolite Signatures of Metabolic Risk Factors and their Longitudinal Changes. J Clin Endocrinol Metab 2016; 101(4): 1779-89.

[15] Tompkins SC, Sheldon RD, Rauckhorst AJ, et al. Disrupting Mitochondrial Pyruvate Uptake Directs Glutamine into the TCA Cycle away from Glutathione Synthesis and Impairs Hepatocellular Tumorigenesis. Cell Rep 2019; 28(10): 2608-19.

[16] Fraga CG, Shigenaga MK, Park JW, Degan P, Ames BN. Oxidative damage to DNA during aging: 8-hydroxy-2'-deoxyguanosine in rat organ DNA and urine. Proc Natl Acad Sci U S A 1990; 87(12): 4533-7.

[17] Esterbauer H, Eckl P, Ortner A. Possible mutagens derived from lipids and lipid precursors. Mutat Res 1990; 238(3): 223-33.

[18] Zhong H, Yin H. Role of lipid peroxidation derived 4-hydroxynonenal (4-HNE) in cancer: focusing on mitochondria. Redox Biol 2015; 4: 193-9.

[19] Toyokuni S, Sagripanti JL. Association between 8-hydroxy-2'-deoxyguanosine formation and DNA strand breaks mediated by copper and iron. Free Radic Biol Med 1996; 20(6): 859-64.

This article is protected by copyright. All rights reserved 
[20] Liu W, Porter NA, Schneider C, Brash AR, Yin H. Formation of 4-hydroxynonenal from cardiolipin oxidation: Intramolecular peroxyl radical addition and decomposition. Free Radic Biol Med 2011; 50(1): 166-78.

[21] Szczesny B, Marcatti M, Ahmad A, et al. Mitochondrial DNA damage and subsequent activation of Z-DNA binding protein 1 links oxidative stress to inflammation in epithelial cells. Sci Rep 2018; 8(1): 914.

[22] Seki S, Kitada T, Yamada T, Sakaguchi H, Nakatani K, Wakasa K. In situ detection of lipid peroxidation and oxidative DNA damage in non-alcoholic fatty liver diseases. J Hepatol 2002; 37(1): 56-62.

[23] Garcia-Martinez I, Santoro N, Chen Y, et al. Hepatocyte mitochondrial DNA drives nonalcoholic steatohepatitis by activation of TLR9. J Clin Invest 2016; 126(3): 859-64.

[24] Gammage PA, Frezza C. Mitochondrial DNA: the overlooked oncogenome? BMC Biol 2019; 17(1): 53.

[25] Dudkina NV, Kouril R, Peters K, Braun HP, Boekema EJ. Structure and function of mitochondrial supercomplexes. Biochim Biophys Acta 2010; 1797(6-7): 664-70.

[26] Andreu AL, Hanna MG, Reichmann H, et al. Exercise intolerance due to mutations in the cytochrome b gene of mitochondrial DNA. N Engl J Med 1999; 341(14): 1037-44.

[27] Lee WS, Sokol RJ. Mitochondrial hepatopathies: advances in genetics and pathogenesis. Hepatology 2007; 45(6): 1555-65.

[28] Meunier B, Fisher N, Ransac S, Mazat JP, Brasseur G. Respiratory complex III dysfunction in humans and the use of yeast as a model organism to study mitochondrial myopathy and associated diseases. Biochim Biophys Acta 2013; 1827(11-12): 1346-61.

This article is protected by copyright. All rights reserved 


\section{FIGURE LEGENDS}

\section{Figure 1}

Electropherograms of the $M T-C Y B$ sequencing from liver samples exhibiting rare pathogenic mutations

A. Heteroplasmic mutations and homoplastic mutations missense mutations. Arrow indicates the location of the base changes; complete details are shown in Supplementary Table 2. B.

Heteroplasmic stop gained mutation. C. Immunohistochemical staining for MT-CYB (mutated and normal protein) in the liver tissue of NAFLD.

\section{Figure 2}

\section{Tissue-damaged mtDNA and circulating metabolites}

A. Analysis of differential liver mtDNA damage at cytochrome b region assessed by semi-long run real-time PCR method in NAFLD patients (morbidly obese $n=65$ : NAFL $n=42$, NASH $n=$ 23; non-morbidly obese $n=127$ : NAFL $n=56$, NASH $n=71$ ). Longitudinal axis stands for the extent of differential mtDNA damage, which was calculated as the inverse of the ratio obtained by normalizing amplification of the long mtDNA fragment with that of the short mtDNA fragment $\left(\Delta C_{\mathrm{p}}\right.$ long fragment/short fragment). Statistical significance of the differences between groups was evaluated using Mann-Whitney U test.

B. Circulating level of metabolites of the Krebs cycle according to the cumulative number of $M T$ $C Y B$ variants $(<10$ and $\geq 10)$. To avoid any bias due to differentially amplified DNA lesions during sequencing, we based the classification of the cumulative number of $M T-C Y B$ variations on the sample of NAFLD overweight/obese patients. Statistical significance of the differences between groups was evaluated using Mann-Whitney U test.

\section{Figure 3}

\section{Immunohistochemical staining for 8-OHdG and 4-hydroxynonenal (4-HNE) in the liver} tissue

8-OHdG and 4-HNE staining in NASH $(\mathbf{A}, \mathbf{D}), \operatorname{NAFL}(\mathbf{B}, \mathbf{E})$, and non-NAFLD $(\mathbf{C}, \mathbf{F})$ patients $(n$ $=8$ per group). Asterisk indicates area shown at higher magnification in the inset $(4 \times$ amplification of the original image). Positive cells show brown staining.

Figure 4

This article is protected by copyright. All rights reserved 


\section{Impact of the cumulative number of $M T-C Y B$ mutations/variation on the whole liver}

transcriptome: significantly up-regulated genes

Volcano plot based upon functional enrichment of significantly up-regulated genes of the global transcriptome. Analysis of differentially expressed pathways was based on the comparison between NAFLD patients with high cumulative number $(\geq 10)$ of MT-CYB mutations/variants versus the reference group (low number of cumulative mutations, i.e., $<10$ ). Enrichment method: ORA (over-representation analysis). Further details are shown in Online Supporting Material.

Figure 5

Impact of the cumulative number of $M T-C Y B$ mutations/variation on the whole liver transcriptome: significantly down-regulated genes

Volcano plot based upon functional enrichment of significantly down-regulated genes of the global transcriptome. Analysis of differentially expressed pathways was based on the comparison between NAFLD patients with high cumulative number $(\geq 10)$ of MT-CYB mutations/variants versus the reference group (low number of cumulative mutations, i.e., $<10$ ). Enrichment method: ORA (over-representation analysis). Further details are shown in Online Supporting Material. 
Table 1

Clinical and biochemical features of NAFLD patients according to the disease status

\begin{tabular}{|c|c|c|c|}
\hline Variables & $\begin{array}{c}\text { NAFL } \\
\text { (nonalcoholic fatty liver) }\end{array}$ & $\begin{array}{c}\text { NASH (nonalcoholic } \\
\text { steatohepatitis) }\end{array}$ & P value \\
\hline \multicolumn{4}{|c|}{ NAFLD overweight / obese patients } \\
\hline Number of subjects & 56 & 71 & - \\
\hline Female (\%) & 53 & 67 & NS \\
\hline Age, years & $55 \pm 11$ & $54 \pm 10$ & NS \\
\hline BMI (body mass index), kg/m² & $31 \pm 5$ & $32 \pm 5$ & NS \\
\hline Fasting plasma glucose, $\mathrm{mg} / \mathrm{dL}$ & $101 \pm 22$ & $123 \pm 43$ & 0.0007 \\
\hline Fasting plasma insulin, $\mu \mathrm{U} / \mathrm{ml}$ & $13 \pm 8$ & $16 \pm 10$ & 0.06 \\
\hline $\begin{array}{l}\text { HOMA-IR (homeostatic model } \\
\text { assessment) index }\end{array}$ & $3.3 \pm 2$ & $4.7 \pm 4.3$ & 0.02 \\
\hline HbA1c & $6.5 \pm 1.5$ & $7.7 \pm 2.5$ & 0.0019 \\
\hline Total cholesterol, mg/dL & $202 \pm 41$ & $208 \pm 49$ & NS \\
\hline HDL-cholesterol, mg/dL & $52 \pm 17$ & $50 \pm 14$ & NS \\
\hline LDL-cholesterol, mg/dL & $124 \pm 36$ & $129 \pm 41$ & NS \\
\hline Triglycerides, mg/dL & $152 \pm 75$ & $173 \pm 105$ & NS \\
\hline ALT, U/L & $35 \pm 17$ & $79 \pm 57$ & 0.0001 \\
\hline AST, U/L & $50 \pm 34$ & $52 \pm 33$ & NS \\
\hline Degree of steatosis $(0-3)$ & $1.6 \pm 0.7$ & $2.2 \pm 0.7$ & 0.0001 \\
\hline Lobular inflammation (0-3) & $0.6 \pm 0.7$ & $1.2 \pm 0.7$ & 0.0001 \\
\hline Hepatocellular ballooning (0-2) & $0.04 \pm 0.2$ & $1 \pm 1$ & 0.0001 \\
\hline Fibrosis Stage & 0 & $1.6 \pm 1.3$ & 0.0001 \\
\hline NAFLD activity score (NAS) & $2.6 \pm 1.3$ & $4.3 \pm 2$ & 0.0001 \\
\hline \multicolumn{4}{|c|}{ NAFLD morbidly obese patients } \\
\hline Number of subjects & 42 & 23 & - \\
\hline Female, $\%$ & 67 & 67 & NS \\
\hline Age, years & $44 \pm 10$ & $46 \pm 11$ & NS \\
\hline
\end{tabular}

This article is protected by copyright. All rights reserved 


\begin{tabular}{|l|c|c|c|}
\hline BMI, kg/m ${ }^{2}$ & $52 \pm 12$ & $48 \pm 11$ & NS \\
\hline Fasting plasma glucose, mg/dL & $108 \pm 34$ & $140 \pm 68$ & 0.01 \\
\hline Fasting plasma insulin, $\mu \mathrm{U} / \mathrm{ml}$ & $13 \pm 7$ & $29 \pm 47$ & 0.03 \\
\hline HOMA-IR index & $3.3 \pm 1.8$ & $6.8 \pm 1.8$ & 0.03 \\
\hline Hemoglobin Alc (glycated & & & $\mathrm{NS}$ \\
hemoglobin) & $6.6 \pm 1.4$ & $185 \pm 54$ & $\mathrm{NS}$ \\
\hline Total cholesterol, mg/dL & $182 \pm 38$ & $40 \pm 7.5$ & 0.01 \\
\hline HDL-cholesterol, mg/dL & $46 \pm 10$ & $132 \pm 45$ & $\mathrm{NS}$ \\
\hline LDL-cholesterol, mg/dL & $127 \pm 27$ & $193 \pm 110$ & 0.022 \\
\hline Triglycerides, mg/dL & $146 \pm 52$ & $44 \pm 20$ & 0.058 \\
\hline ALT (alanine aminotransferase), & $33 \pm 23$ & & 0.06 \\
\hline U/L & $25 \pm 17$ & $33 \pm 16$ & 0.0001 \\
\hline AST (aspartate & $1.6 \pm 0.8$ & $1.5 \pm 0.9$ & 0.0001 \\
\hline aminotransferase), U/L & $0.3 \pm 0.6$ & $1 \pm 0.6$ & 0.0001 \\
\hline Degree of steatosis, \% & $0.16 \pm 0.4$ & $2.2 \pm 0.7$ & 0.0037 \\
\hline Lobular inflammation (0-3) & $0.02 \pm 0.2$ & & 0.3 \\
\hline Hepatocellular ballooning (0-2) & $2 \pm 1.3$ & & \\
\hline Fibrosis Stage & & & \\
\hline NAFLD activity score (NAS) & & & \\
\hline
\end{tabular}

Results are expressed as mean $\pm \mathrm{SD}$. P-value stands for statistical significance using the MannWhitney $U$ test, except for female/male proportion that $\mathrm{p}$-value stands for statistical significance using the Chi-square test.

This article is protected by copyright. All rights reserved 
A
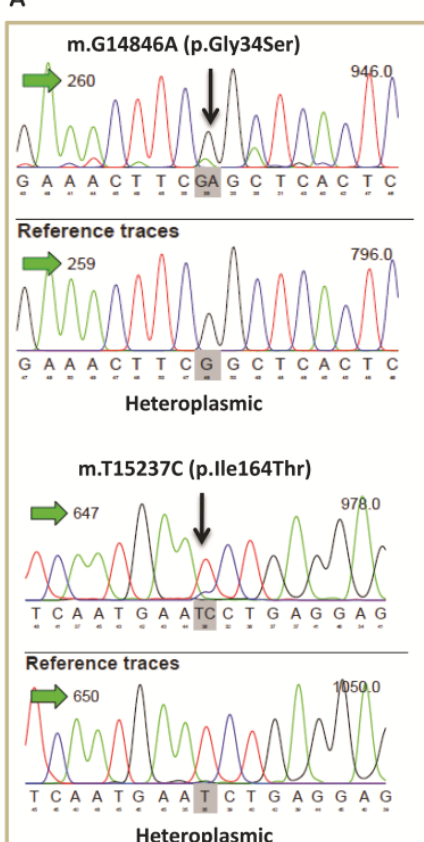

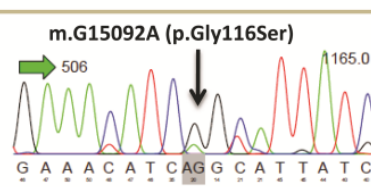

Reference traces

$\Rightarrow 505 \quad 1110.0$

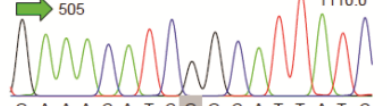

GAAACATCGG CA T T A T C

Heteroplasmic
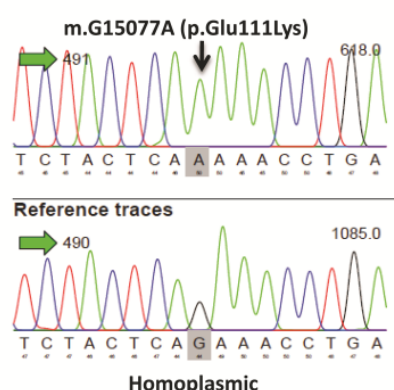

Homoplasmic

B

m.G15242A (p.Gly166Ter) Stop gained $\Rightarrow 655$ mandwa GA A T C T G AGAG AGG C TAC

Reference traces

$\Rightarrow 655$ MNAV GAATCT GAGGAGGCTAC Heteroplasmic

C

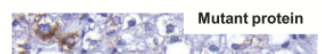

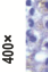

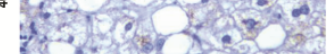

ringenas

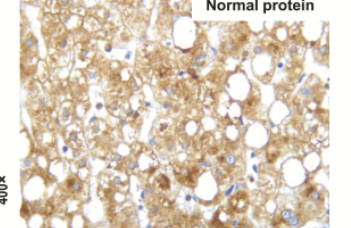

joim_13147_f1.tif

This article is protected by copyright. All rights reserved 
A

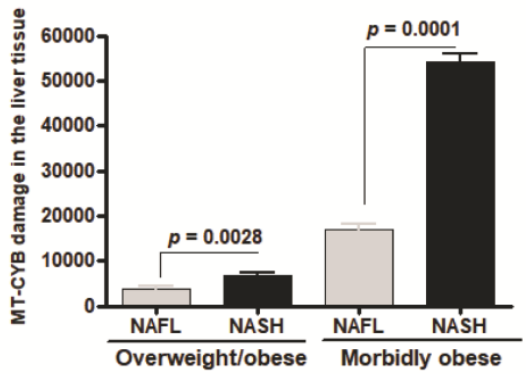

B

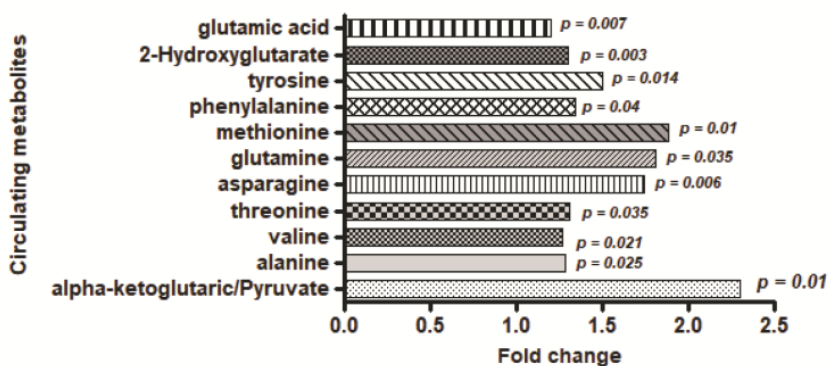

joim_13147_f2.tif

This article is protected by copyright. All rights reserved 

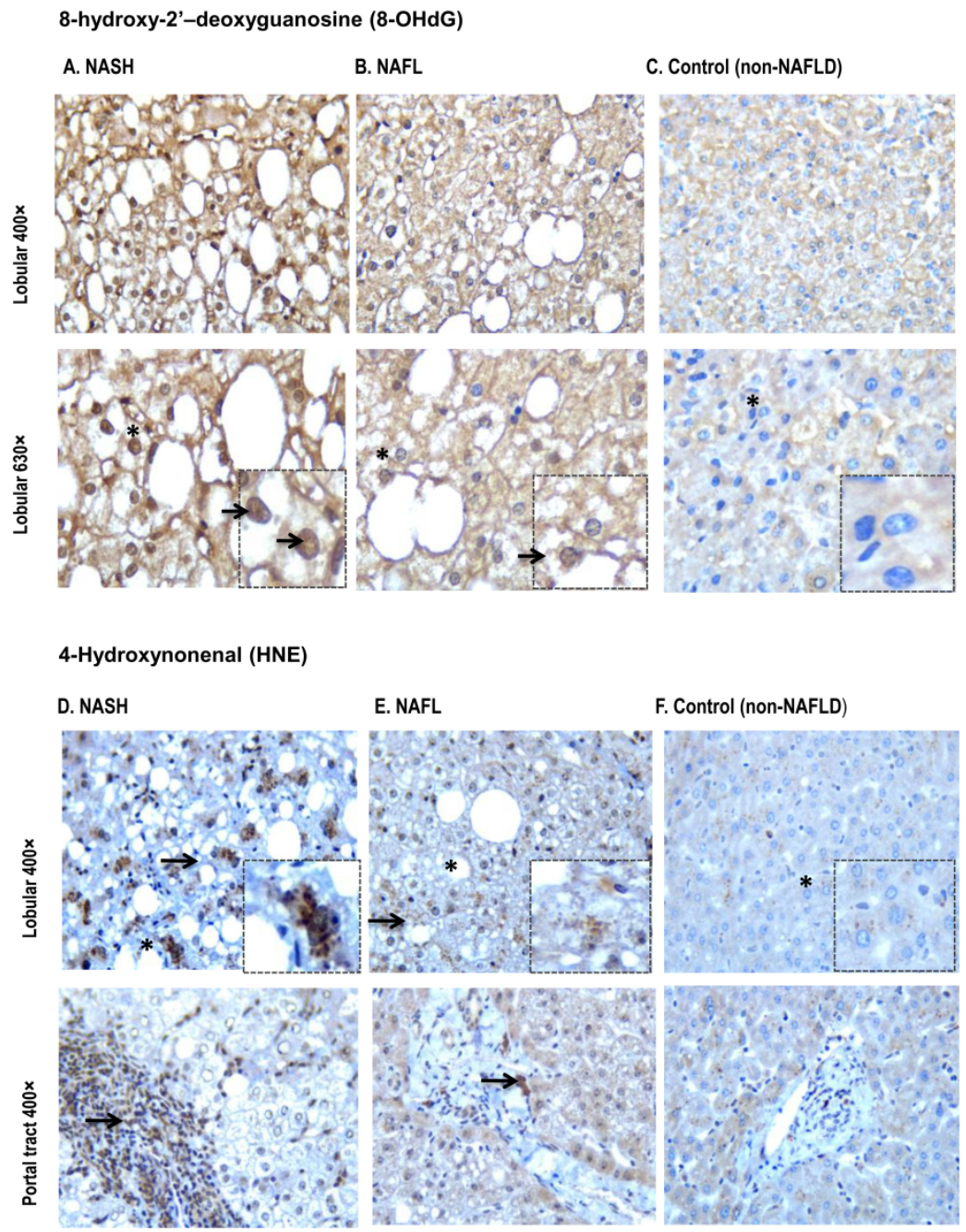

joim_13147_f3.tif 
Global transcriptome in NAFLD patients with high cumulative number $(\geq 10)$ of $M T$-CYB mutations/variants: ORA analysis based upon significantly up-regulated genes

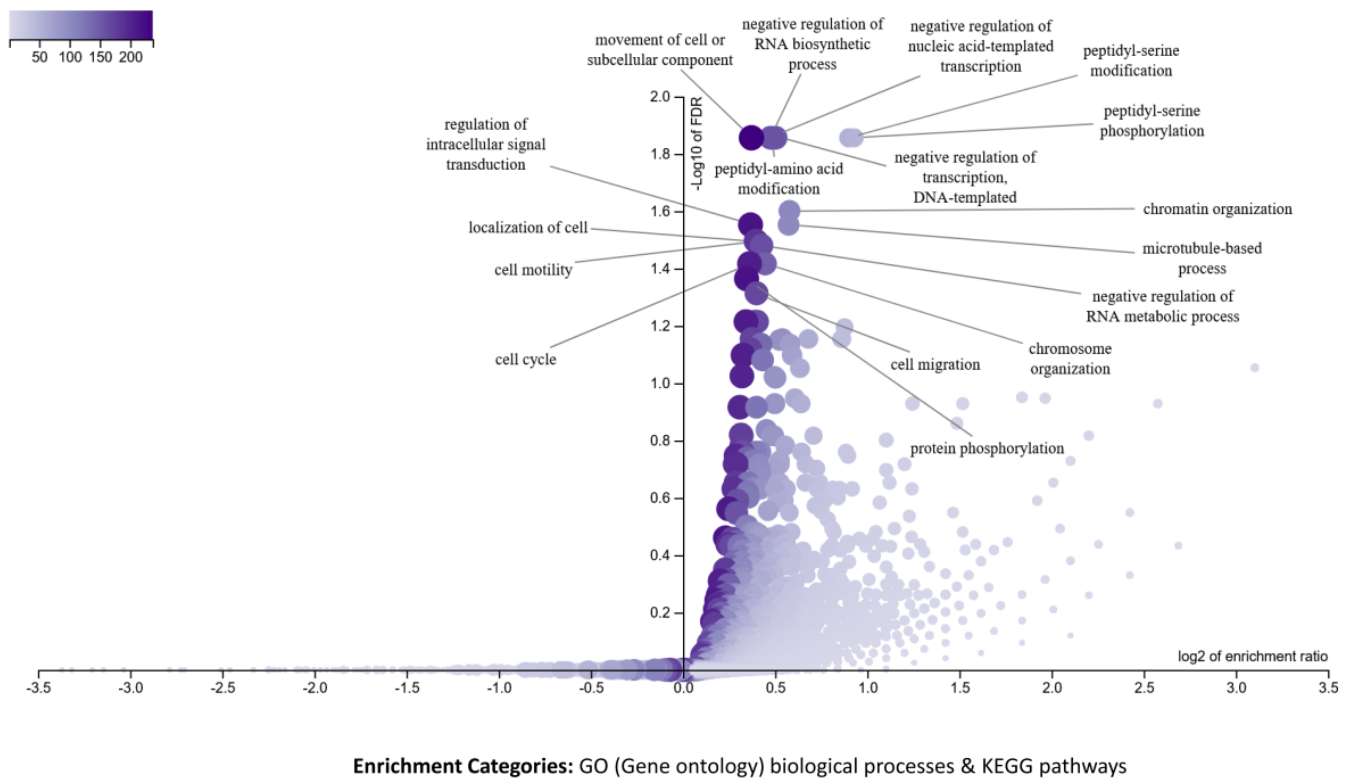

joim_13147_f4.tif

This article is protected by copyright. All rights reserved 
Global transcriptome in NAFLD patients with high cumulative number $(<10)$ of MT-CYB mutations/variants: ORA analysis based upon significantly down-regulated genes

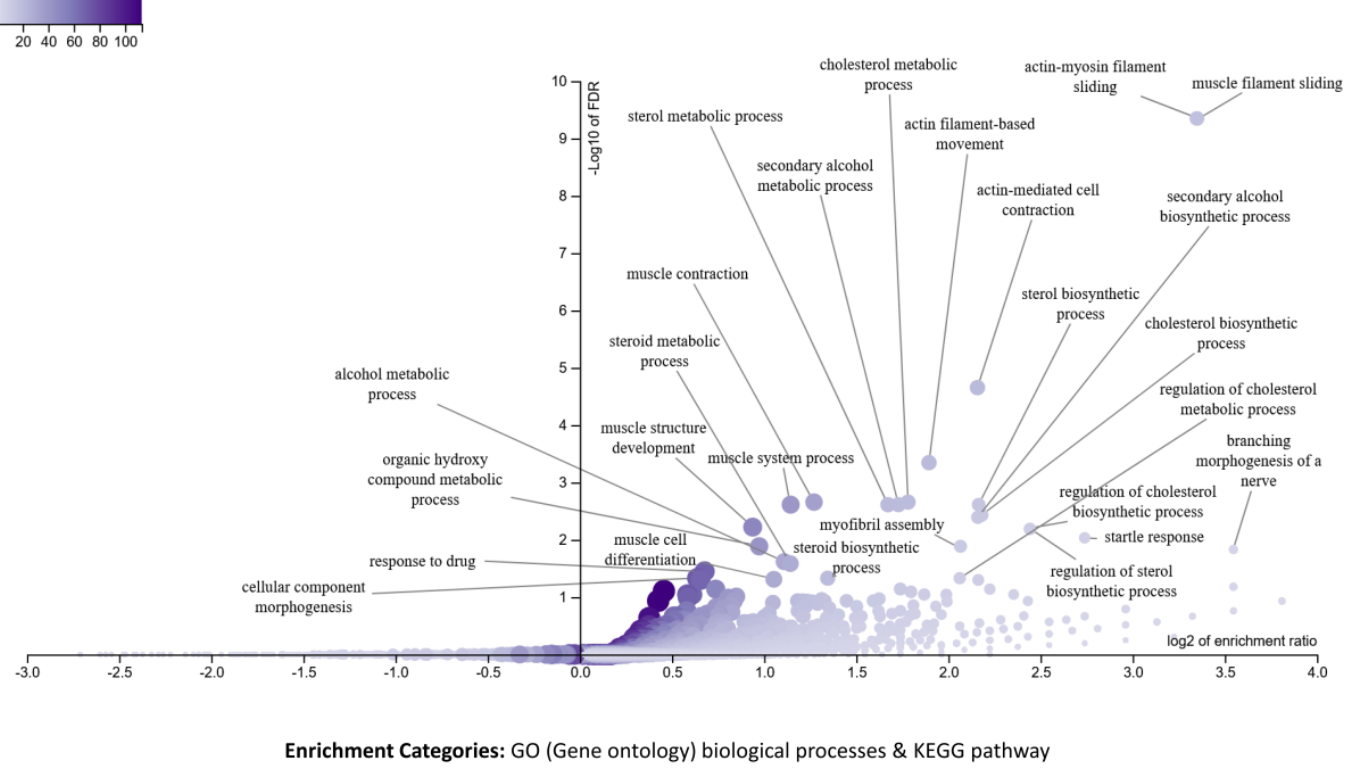

joim_13147_f5.tif

This article is protected by copyright. All rights reserved 